\title{
The cryomechanical design of MUSIC - A novel imaging instrument for millimeter-wave astrophysics at the Caltech Submillimeter Observatory
}

\author{
Matthew I. Hollister ${ }^{a}$, Nicole G. Czakon ${ }^{a}$, Peter K. Day ${ }^{b}$, Thomas P. Downes $^{a}$, Ran Duan $^{a}$, \\ Jiansong $\mathrm{Gao}^{c}$, Jason Glenn ${ }^{d}$, Sunil R. Golwala ${ }^{a}$, Henry G. LeDuc ${ }^{b}$, Philip R. Maloney ${ }^{d}$, \\ Benjamin A. Mazin ${ }^{e}$ Hien Trong Nguyen $^{b}$, Omid Noroozian ${ }^{a}$, Jack Sayers ${ }^{b}$, James Schlaerth $^{d}$, \\ Seth Siegel ${ }^{a}$, John E. Vaillancourt ${ }^{f}$ Anastasios Vayonakis ${ }^{a}$, Philip Wilson ${ }^{b}$ and Jonas \\ Zmuidzinas $^{a}$ \\ ${ }^{a}$ California Institute of Technology, Pasadena, CA 91125; \\ ${ }^{b}$ Jet Propulsion Laboratory, Pasadena, CA 91109; \\ ${ }^{c}$ National Institute of Standards and Technology, Boulder, CO 80305; \\ ${ }^{d}$ CASA, University of Colorado, Boulder, CO 80309; \\ ${ }^{e}$ University of California, Santa Barbara, CA 93106; \\ fSOFIA/USRA, NASA Ames Research Center, Moffet Field, CA 94035
}

\begin{abstract}
MUSIC (Multicolor Submillimeter kinetic Inductance Camera) is a new facility instrument for the Caltech Submillimeter Observatory (Mauna Kea, Hawaii) developed as a collaborative effect of Caltech, JPL, the University of Colorado at Boulder and UC Santa Barbara, and is due for initial commissioning in early 2011. MUSIC utilizes a new class of superconducting photon detectors known as microwave kinetic inductance detectors (MKIDs), an emergent technology that offers considerable advantages over current types of detectors for submillimeter and millimeter direct detection. MUSIC will operate a focal plane of 576 spatial pixels, where each pixel is a slot line antenna coupled to multiple detectors through on-chip, lumped-element filters, allowing simultaneously imaging in four bands at $0.86,1.02,1.33$ and $2.00 \mathrm{~mm}$.

The MUSIC instrument is designed for closed-cycle operation, combining a pulse tube cooler with a two-stage Helium-3 adsorption refrigerator, providing a focal plane temperature of $0.25 \mathrm{~K}$ with intermediate temperature stages at approximately 50, 4 and $0.4 \mathrm{~K}$ for buffering heat loads and heat sinking of optical filters. Detector readout is achieved using semi-rigid coaxial cables from room temperature to the focal plane, with cryogenic HEMT amplifiers operating at $4 \mathrm{~K}$. Several hundred detectors may be multiplexed in frequency space through one signal line and amplifier.

This paper discusses the design of the instrument cryogenic hardware, including a number of features unique to the implementation of superconducting detectors. Predicted performance data for the instrument system will also be presented and discussed.
\end{abstract}

Keywords: MUSIC, Submillimeter detector array, Cryogenics, Pulse tube cooler, Mechanical design

\section{INTRODUCTION}

The development of large-format instruments with arrays of incoherent detectors for astronomy at millimeter and submillimeter wavelengths has proved to be extremely challenging. However, in the last few years considerable success has been realized through the use of transition-edge sensor (TES) bolometers ${ }^{1}$ multiplexed with SQUID arrays, ${ }^{2}$ with instruments such as SCUBA-2 on the James Clerk Maxwell Telescope ${ }^{3}$ producing first results. However, focal planes of TES bolometers and SQUIDs present considerable technical challenges in implementation. For example, although SQUIDs allow a degree of multiplexing, considerable wire counts are still required

Further author information: (Send correspondence to M.H.)

M.H.: E-mail: matt.hollister@physics.org

Millimeter, Submillimeter, and Far-Infrared Detectors and Instrumentation for Astronomy V, edited by Wayne S. Holland, Jonas Zmuidzinas, Proc. of SPIE Vol. 7741, 77411L

(C) 2010 SPIE $\cdot$ CCC code: $0277-786$ X/10/\$18 $\cdot$ doi: $10.1117 / 12.856780$

Proc. of SPIE Vol. 7741 77411L-1 
Table 1: Mass of hardware at each stage of the instrument. Need to confirm these numbers.

\begin{tabular}{lcc}
\hline Stage & Temperature & Mass / kg \\
\hline Vacuum jacket & $300 \mathrm{~K}$ & 95 \\
First stage & $50 \mathrm{~K}$ & 40 \\
Second stage & $4 \mathrm{~K}$ & 85 \\
Third stage & $0.4 \mathrm{~K}$ & 6 \\
Fourth stage & $0.25 \mathrm{~K}$ & 4 \\
\hline Total & & 230 \\
\hline
\end{tabular}

for large arrays, with the associated conduction loads from room temperature, and power dissipation in the cold electronics, often at temperatures below $1 \mathrm{~K}$. Further to this, there is the inherent sensitivity of SQUIDs to magnetic fields to consider, leading to considerable challenges in the design of effective shielding to reduce the magnetic environment at the devices to an acceptable level.

More recently, a new type of device suitable for direct detection at millimeter and submillimeter wavelengths, the microwave kinetic inductance detector (MKID), has started to generate interest. MKIDs are superconducting pair breaking detectors, as opposed to thermal detectors such as TES bolometers, in which the absorption of optical power breaks the paired electrons in the superconductor. The MKID is a thin superconducting film, fabricated as part of an inductive-capacitive resonance circuit. The absorption of optical power causes a shift in both the frequency and quality factor of the resonance. Multiple resonators with stepped resonant frequencies may be coupled to a common microwave feedline to produce an array of detectors multiplexed in the frequency domain. Further details of the physics of MKIDs is beyond the scope of this paper, but may be found elsewhere. ${ }^{4}$

A key advantage of the use of MKIDs at millimeter wavelengths is that an array architecture may be realized in which multiple detectors are coupled through lithographic filters to a phased array antenna, resulting in pixels that simultaneously image in multiple bands. Recently, a collaboration between the University of Colorado, Boulder, the California Institute of Technology, NASA's Jet Propulsion Laboratory and the University of California, Santa Barbara, have been working towards the deployment of a camera based on this array architecture for use at the Caltech Submillimeter Observatory (CSO), Mauna Kea, Hawaii. This instrument is the Multicolor Submillimeter kinetic Inductance Camera, or MUSIC (formerly the MKID Camera). Details of both the general project $^{5}$ and latest detector results ${ }^{6,7}$ may be found elsewhere in these proceedings. This paper deals with the cryomechanical design of MUSIC. The main features of the instrument design will be described, including details of predicted low temperature performance.

\section{TOP LEVEL DESCRIPTION}

MUSIC is intended as a wide field camera, providing a $>14$ arcminutes field of view from the Cassegrain focus of the CSO. The instrument focal plane has a total of 576 spatial pixels divided between 8 subarrays, with each pixel imaging simultaneously in four bands at $0.86,1.02,1.33$ and $2.00 \mathrm{~mm}$ matched to the atmospheric transmission windows. A more detailed discussion of the optical design and predicted performance of the instrument may be found elsewhere in these proceedings. ${ }^{8}$

The MUSIC focal plane must be operated at $<300 \mathrm{mK}$ in order to achieve the required sensitivity to astronomical sources. The cryogenic system for MUSIC can broadly be divided into two subsystems. The first and second stages of the instrument are cooled to 50 and $4 \mathrm{~K}$ by a Cryomech* PT415 pulse tube cooler. These stages provide buffering of radiative and conductive loads, and in the case of the 4-K stage, heat sinking for low temperature amplifiers. Two further temperature stages are cooled to 360 and $250 \mathrm{mK}$ by a Chase Cryogenics ${ }^{\dagger}$ two-stage ${ }^{3} \mathrm{He}$ adsorption refrigerator. The third stage provides further buffering of conductive loads, while the MKID

\footnotetext{
${ }^{*}$ http://www.cryomech.com

${ }^{\dagger}$ http://www.chasecryogenics.com
} 


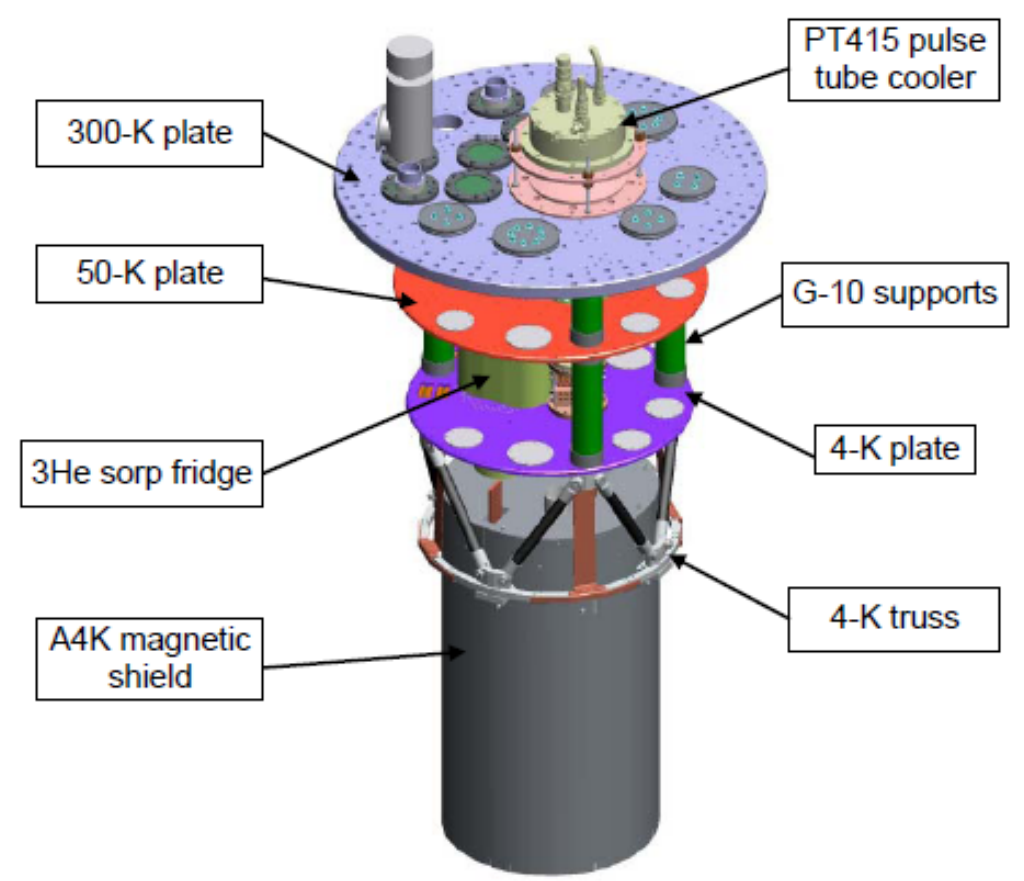

Figure 1: Computer general model cross section of the cryostat, with the major structural features indicated. See text for further discussion.

focal plane module is mounted at the fourth stage. The basic MUSIC 4-K cryostat was originally designed and constructed by High Precision Devices Inc., but has been subsequently modified to accommodate the camera cold hardware. Table 1 summarizes the mass of hardware at each temperature stage of the instrument.

A feature of the use of superconducting devices for astronomical instruments is the need to control the magnetic field environment at the detectors, since changes in the local magnetic field, for example, due to moving the telescope during observations, will appear in the detector output. Although the raw sensitivity of MKIDs to changes in the magnetic field environment is comparable to that of newer generation SQUID-based readouts, work in related fields suggests that the frequency shift of superconducting resonators in response to a changing magnetic field falls of the the square of the field, ${ }^{9}$ rather than linearly as for a SQUID. ${ }^{10}$ For the MUSIC cryostat, a multistage magnetic shield solution was developed using an iterative process of finite-element analysis. The adopted solution consists of a dual-layer shield of high permeability material at $4 \mathrm{~K}$, combined with a superconducting shield surrounding the focal plane. In addition to this, it is intended that a superconducting backshort will be integrated with the focal plane. The details of the finite-element analysis are beyond the scope of this work, but the overall predicted attenuation for this shielding design is 5000, which, based on the measured unshielded response of the MKIDs at the CSO, will be sufficient to reduce the detector response to a negligible level. Details of the design of the shielding stages are included in the discussion in the following sections.

\section{VACUUM VESSEL}

The MUSIC vacuum vessel is composed of four sections fabricated from Aluminium 6061-T6 alloy, and joined via demountable bolted flanges. The cryostat top plate includes all of the ports into the vacuum space, including the mounting flange for the pulse tube cooler cold head. This plate also acts as the mounting point for the supports of the internal cold structures. Two cylindrical sections with wall thicknesses of .160" and inside diameters of 21.6", forming a vacuum space 53.5" in length. The vacuum window assembly, with a 15" ultra-high molecular weight polyethylene window providing a 12" clear aperture, mounts on the lower end plate of the vacuum vessel. This lower flange also provides the interface to the warm relay optics. 


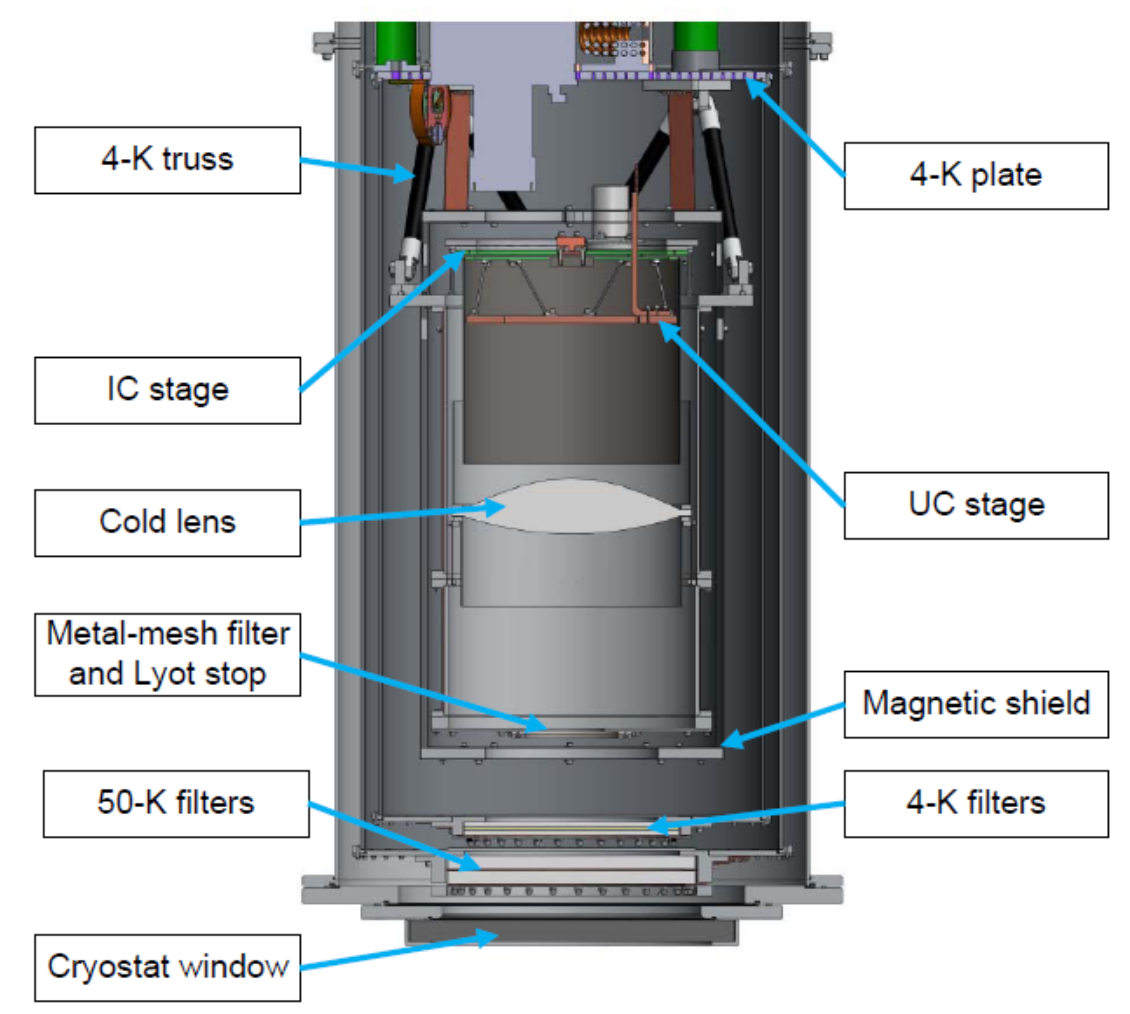

Figure 2: Cross section of the cryostat cold hardware and optics, with the key features indicated. For more details, see discussion in text.

\section{50-K AND 4-K STAGES}

The first and second stages of the cryostat are cooled by a Cryomech PT415 pulse tube cooler. The cooler nominally provides $1.35 \mathrm{~W}$ of heat uplift at $4 \mathrm{~K}$ and $40 \mathrm{~W}$ at $45 \mathrm{~K}$. The cold head includes a remote motor and bellows assembly at the vacuum flange to reduce vibrations, further supplemented with flexible wicks of soldered copper braids between the cryostat first and second stage plates and the pulse tube heat exchangers.

A view of the instrument cryostat solid model is shown in Fig. 1, with the major features indicated. The first stage plate, at a nominal temperature of $50 \mathrm{~K}$, is a 20.375" diameter, 0.375 "-thick Aluminium 6061 plate, interfacing to a cylindrical radiation shield, 45" in length. The walls of the shield are fabricated from .190" thick Aluminium 1100-O to minimize the temperature gradient along the shield, welded to flanges of Aluminium 6061. The end plate of the shield is a .250"-thick Aluminium 6061 plate that serves as a mount for the 50-K filter assembly (see discussion below). A multilayer insulation blanket, consisting of 20 layers of .001" aluminized mylar spaced with spun polyester is attached to the outside of the $50-\mathrm{K}$ shield to reduce the radiation load from the vacuum jacket wall.

The $50-\mathrm{K}$ stage is attached to the $300-\mathrm{K}$ plate by three vertical G-10 tubes with outside diameter 2", $\frac{1}{16}$ " wall thickness, and 4.65" long. The 4-K stage is suspended from the $50-\mathrm{K}$ plate by three similar tubes, 6.75 " long. As is discussed in more detail below, vertical tubes produce a support structure that is good for loads parallel to the long axis of the structure, but weak for lateral loads. Finite-element analysis of the supports had indicated acceptable deflection under the expected operational loading.

The 4-K cold plate is gold-plated ETP copper, 18.625" diameter and .45" thickness. The 4-K plate serves as the mounting for the ${ }^{3} \mathrm{He}$ sorp fridge, in addition to a second radiation shield. The 4-K shield is also fabricated from an Aluminium 6061 cylinder, with a 0.125" wall, with welded Aluminium 6061 flanges. The shield is 35" in length, with additional infrared blocking filters at the entrance aperture. 


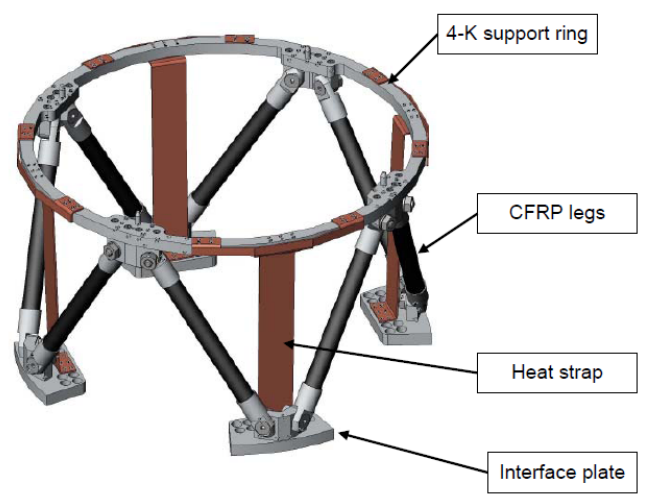

Figure 3: Main 4-K truss assembly.

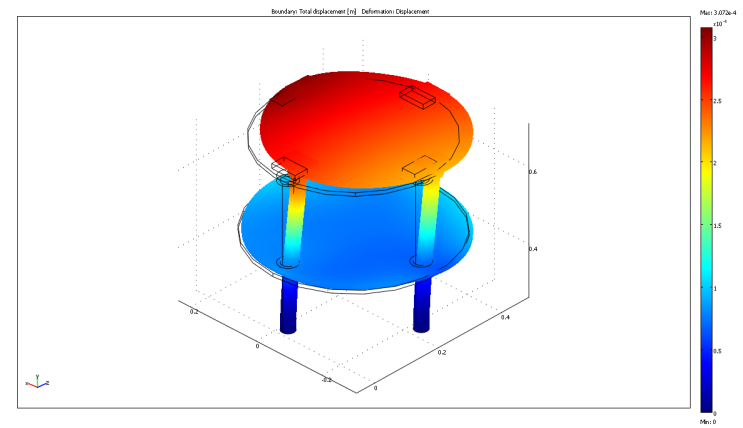

Figure 5: Finite-element analysis of deflection in the main G-10 cryostat support structure with a load of $100 \mathrm{~kg}(1.05 \mathrm{~g})$ at an angle of 45 degrees from vertical.

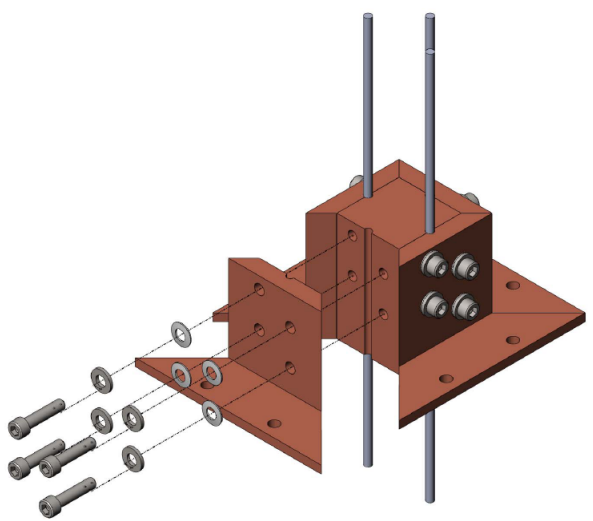

Figure 4: CAD model of one of four heat sinks for the semirigid coaxial cables at the $50-\mathrm{K}$ plate. Three coaxial cables are shown in the imate.

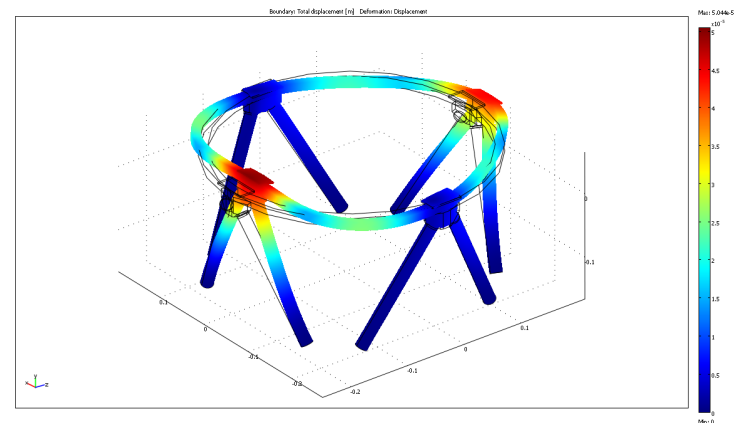

Figure 6: Finite-element analysis of deflection in the main $4-\mathrm{K}$ truss structure with a load of $100 \mathrm{~kg}$ $(1.05 \mathrm{~g})$ at an angle of 45 degrees from vertical.

Infrared filtering in the MUSIC cryostat is provided by a combination of capacitive metal mesh filters ${ }^{11}$ produced by QMC Instruments, ${ }^{\ddagger}$ and PTFE dielectric filters. At the 50 -K shield, the radiative load from the $300-\mathrm{K}$ vacuum window is initially reduced by a pair of QMC polypropylene shaders, followed by two .625"-thick PTFE discs. The QMC shaders are used to minimise heating of the teflon filters at the 50-K shield due to the radiative load from the $300 \mathrm{~K}$ vacuum window. The PTFE filters are separated by a small air gap, and are well heat sunk to the top of the 50-K radiation shield with copper heat straps. The clear aperture at the $50-\mathrm{K}$ shield is 10 ". At the 4-K shield, a pair of .25"-thick PTFE discs sandwich a .125"-thick fluorogold disc, with the stack again well heat sunk with a copper strap. Antireflection coatings, tuned for a wavelength of $1 \mathrm{~mm}$, are provided by Porex sheets bonded to the filter surfaces using LDPE as an adhesive. Inside the 4-K cold space, the edge of the filter pass band is defined by a $13.5 \mathrm{~cm}^{-1}$ QMC metal mesh low pass filter mounted at the cold stop.

The major cold hardware assembly is mounted to the 4-K cold plate via a octopod truss formed by .75" diameter carbon fiber reinforced polymer (CFRP) rods (produced by Diversified Structural Composites) bonded into aluminium end caps with Stycast 2850FT epoxy. A computer model image of this truss is shown in Fig. 3. The rod assemblies are bolted to an aluminium ring that provides further mounting points for heat straps, in addition to constraining the trusses to improve the stiffness of the structure. This aluminium ring is heat sunk to the $4-\mathrm{K}$

\footnotetext{
${ }^{\ddagger}$ http://www.terahertz.co.uk
} 
Table 2: Predicted heat loads on the first and second stage of the cryostat, in decreasing order of magnitude at the first stage.

\begin{tabular}{lcc}
\hline Element & First stage load / W & Second stage load / W \\
\hline Radiation & 10 & 0.027 \\
Optical power & 3 & 0.1 \\
Supports & 0.95 & 0.066 \\
Signal coax & 0.281 & 0.038 \\
Service wiring & 0.188 & 0.107 \\
HEMT amplifiers & $\ldots$ & 0.25 \\
Sub-kelvin fridge & $\ldots$ & 0.008 \\
\hline Total & 14.42 & 0.596 \\
\hline
\end{tabular}

cold plate via four ETP copper heat straps. Although this truss is not intended to be thermally isolating, CFRP makes an excellent choice for structural materials due to the high ratio of the the material stiffness (the Young's modulus of CFRP is twice that of aluminium alloys) to both cost and mass. It was a key design requirement for this truss that the deflection under gravity be considerably less than the deflection of the main G-10 cold plate supports, since these vertical support tubes will dominate the overall flexure in the cryostat. Finite-element analysis was used to determine the deflection of the G-10 supports under load, and to validate the design of the other truss structures. Example results from the FEA process are shown in Fig. 5 for the G-10 supports, and in Fig. 6 for the 4-K truss structure. In both cases, the deflection under horizontal loading are shown (the overall load direction is at 45 degrees to the vertical, since this is the maximum tilt the cryostat will experience in normal operation), since this component will be greater than the deflection under vertical loading. It may be seen that the vertical G-10 trusses deflect significantly under lateral loading (the maximum deflection in these simulations is $300 \mu \mathrm{m}$ ). The carbon fiber $4-\mathrm{K}$ truss, conversely, provides an extremely stiff mount, with predicted deflections from FEA of $50 \mu \mathrm{m}$ under horizontal loading. The design principle of using aluminium and CFRP structures to minimize mass and flexure has been used throughout the cryostat design, with key cooling paths supplemented with copper heat straps.

Attached to the 4-K truss assembly is a second aluminium ring that is the mounting point for both the cold optics assembly and the sub-kelvin hardware, discussed in the next section. This hardware is enclosed by a high-permeability magnetic shield fabricated from two concentric cylinders of Amumetal 4K, a low temperature material produced for the Amuneal Manufacturing Corp. that is replacing the more commonly used Cryoperm-10 high-permeability shielding alloy. The cylinders are fabricated from .040"-thick material, with a spacing between the two cylinders of .30", built around an aluminium frame that gives structural support. The shield is fabricated in two parts, allowing the upper section to be removed to access the enclosed hardware. Additional copper straps are used to ensure good heat sinking of the top of the shield to the 4-K truss, primarily to reduce the cool down time rather than to minimize the temperature gradient.

The cold optics assembly is a two part Aluminium 6061 cylinder that defines the position of the cold stop and provides a mount for the cold lens. The inside of the cylinder is blackened with carbon-loaded epoxy for stray light control. The top plate of the cylinder forms the cold Lyot stop, consisting of a G-10 disc with a 3" circular hole machined with a knife edge. Also mounted at the cold stop is the metal mesh low pass filter discussed previously. The 11"-diameter cold lens, fabricated from ultra-high molecular weight polyethylene, is mounted inside the optics assembly cylinder. The lens is suspended on a series of 8 copper tab flexures that provide both heat sinking and mechanical support. The 1/16"-thick tabs allow flexure radially to account for thermal contraction of the plastic lens, but provide a rigid support against motion in all other directions. Blackened baffles extend from the lens to the cold stop, and from the lens towards the focal plane, for further control of stray light. Heat sinking of the lens flange and cold stop through the aluminium wall of the cylinder is supplemented with ETP copper straps. 


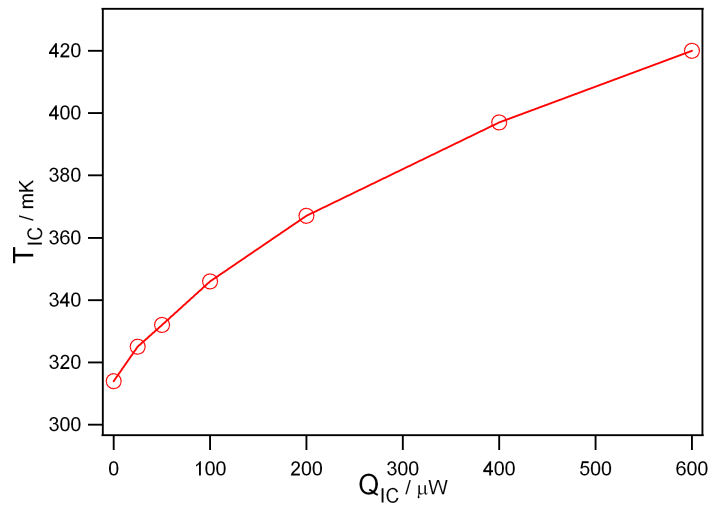

Figure 7: Cooling capacity of the intercooler (IC) still.

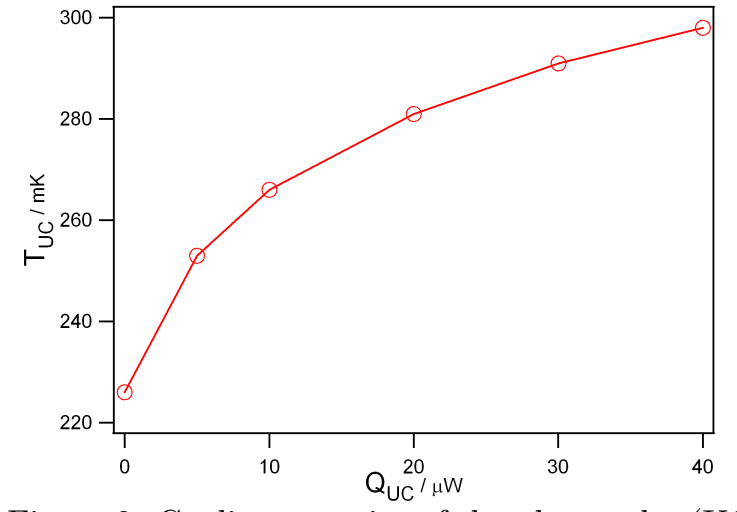

Figure 8: Cooling capacity of the ultracooler (UC) still.

Signal cabling to the $4-\mathrm{K}$ space consists of 8 pairs of semi-rigid coaxial cables. Between the room temperature flange and the $4-\mathrm{K}$ plate, these coaxs are made from single lengths of 0.086 " stainless steel cable terminated in welded SMA connectors. The cables are heat sunk at the 50-K plate by clamping between gold-plated copper blocks, with four coaxial cables clamped into a single assembly that is then mounted to the 50-K cold plate (see Fig. 4). The stainless steel coaxs attached to hermetic feedthroughs at the $300-\mathrm{K}$ plate, and bulkhead connectors heat sunk at the 4-K cold plate. On the cold side of the 4-K plate, 8 HEMT low-noise amplifiers (one per coax pair) are mounted on copper brackets. The HEMT bias connections are provided by a cable harness consisting of 32.005 " manganin wires for the HEMT gates (2 wire pairs to each amplifier for redundancy) and 32.005 " copper wires to the amplifier drain and common ground, again with paired wires for redundancy.

Table 2 summarizes the calculated contributions of the various components to the thermal loads on the first and second stage of the cryostat. As would be expected, the heat loads on the first stage are dominated by the radiative loading from the $300 \mathrm{~K}$ vacuum shell and the load in the optical path from the vacuum window. The G-10 structural supports for the cold hardware contribute a further $950 \mathrm{~mW}$ of heat. The conduction calculation for the 16 signal coax lines assumes that only the outer conductors are sunk at the first stage, which is effectively the case since the coax cables are only clamped to the $50-\mathrm{K}$ cold plate. The total calculated load on the first stage of the cryostat is $14.4 \mathrm{~W}$, which from performance figures for the PT415 pulse tube published by Cryomech is equivalent to a temperature at the first stage of the PTC of $34 \mathrm{~K}$.

The total heat load on the second stage of the cryostat is calculated to be approximately $600 \mathrm{~mW}$, dominated by the power dissipation of the 8 HEMT LNAs in normal operation. The power dissipation of individual HEMTs varies dependant upon the exact operating point of the the particular amplifier, but a figure of $\sim 31 \mathrm{~mW}$ per LNA is taken as an upper value to allow for margin in operation. Since the HEMTs dominate the heat loads at the second stage, it is likely that the amplifiers will be powered down to allow additional overhead on the PTC cooling capacity during recycling of the ${ }^{3} \mathrm{He}$ refrigerator. From the published performance figures for the pulse tube cooler, it is expected that the cold head second stage will operate at $3.5 \mathrm{~K}$.

\section{SUB-KELVIN STAGES}

The third and fourth stages of the instrument are cooled by a two stage ${ }^{3} \mathrm{He}$ adsorption refrigerator. The cooling capacities of the intercooler (IC) and ultracooler (UC) stills of the refrigerator are shown in Figs. 7 and 8 , respectively. The total heat uplift for the IC still has been measured to be $\sim 21 \mathrm{~J}$, with $\sim 3.2 \mathrm{~J}$ available on the $\mathrm{UC}$ still. A CAD image of the layout of the third and fourth stage hardware is shown in Fig. 9.

The IC cold head is used to buffer the conductive loads from mechanical supports and the signal coax lines between the 4-K stage and focal plane. There are two separate cooling paths to the IC cold head. The first is connected to a stage that directly buffers the conductive loading from structural supports. This stage is fabricated from Aluminium 5052, an alloy with a depressed superconducting transition temperature. This stage is supported from the main 4-K structure by 8 trusses composed of .125" diameter CFRP rods epoxied to Aluminium 6061 


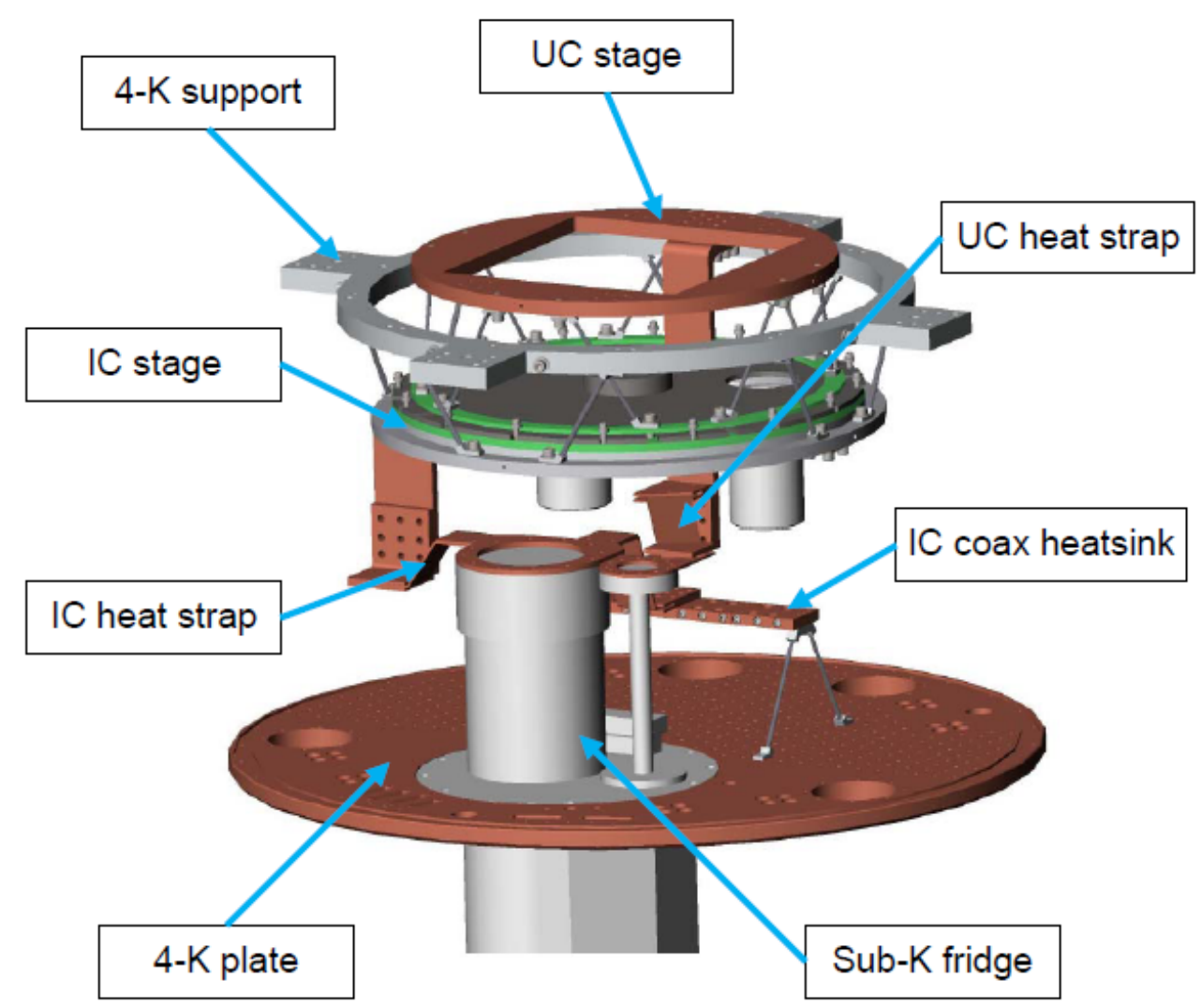

Figure 9: Layout of the sub-kelvin stages of the cryostat, with the key features indicated. For more details, see discussion in text.

end pieces. The CFRP used for these isolating supports is Graphlite, a pultruded rod product produced by Aviva Sports Composites. ${ }^{12}$ The IC stage also serves as a mount for a superconducting shield fabricated from .039"thick niobium, formed into a welded tube. The cylindrical section of the shield is demountable to allow access to the internal structures. The shield sits on a G-10 ring that isolates the bulk structure from the aluminium stage. Cooling is then via a short ETP copper wick to ensure that magnetic flux is expelled from the superconductor during cooling. Although the superconducting shield could have been sunk at the second stage of the cryostat (the transition temperature of niobium being $\sim 9 \mathrm{~K}$ ), the shield was mounted at the IC stage for mechanical convenience.

Also cooled by the IC stage of the ${ }^{3} \mathrm{He}$ fridge is a separate copper platform used to heat sink the semirigid coaxial cables. This stage is isolated from the $4-\mathrm{K}$ cold plate by four legs constructed from .098" diameter Graphlite CFRP. From the 4-K bulkhead feedthroughs, and between the coax heatsink and the HEMT amplifiers, the coaxs are .064" niobium-titanium superconducting cables approximately 8 " in length. The coax lines terminate at a fixed attenuator that is clamped to the the copper stage, providing heat sinking of both the inner and outer conductor of the coax cable.

The UC stage is a gold-plated ETP copper disc, 8" in diameter, supported from the IC stage using 8.098 " diameter Graphlite legs. The stage is completely enclosed by the niobium superconducting shield can. The focal plane module (FPM) interfaces directly to the copper stage via a bolted joint. Although the detailed design has yet to be finalized, the FPM will comprise a self-contained unit that can be removed from the cryostat as a single component. Within the module, 8 detector tiles are mounted using Beryillium Copper spring clamps, allowing for movement during thermal contraction. The tiles form a mosaic covering the area of the focal plane, approximately $5.5 \times 5$ ", with spacings between individual tiles of $<1$ pixel. Crystal quartz antireflection tiles 
Table 3: Calculated heat loads on the sub-kelvin stages of the cryostat. Need to fix the fourth stage loads.

\begin{tabular}{lcc}
\hline Element & Third stage load $/ \mu \mathrm{W}$ & Fourth stage load $/ \mu \mathrm{W}$ \\
\hline Supports to stage & 60.9 & 0.122 \\
Supports to coax heat sink & 15.5 & $\mathrm{n} / \mathrm{a}$ \\
Service wiring & 0.2 & $3 \times 10^{-4}$ \\
Signal coax & 61.0 & 0.026 \\
Radiation & 0.4 & 0.2 \\
Optical power & $\mathrm{n} / \mathrm{a}$ & 2.0 \\
\hline Total & 138 & 2.348 \\
\hline
\end{tabular}

tuned to $\lambda=1 \mathrm{~mm}$ are placed under the detector tiles, and are held in place by the force of the spring clamps. Additional heat sinking of the detector tiles to the metal of the box is provided by gold wire bonds around the tile perimeter. The assembled FPM will also incorporate a niobium cover that forms both a superconducting backplane for the array, and an optical backshort to increase the efficiency of the radiation absorption by the detectors. Connections to the focal plane from the IC heat sink are also NbTi coaxial cables, 10" in length, terminating at the copper UC stage with blindmate connectors. ${ }^{\S}$ The use of blindmate connectors allows the FPM to be connected and removed from the cryostat without having to individually mate SMA connections.

The calculated heat loads on the IC and UC cold heads are summarized in Table 3. At the IC stage, $55 \%$ of the $138 \mu \mathrm{W}$ total heat load comes from the CFRP structural supports from the 4-K stage of the cryostat, with a further $44 \%$ from the NbTi coaxial cables. Note that due to the structural arrangement of the cold hardware, there is effectively no coupling between the IC stage and the optical beam. From the measured cooling capacity of the IC still, it is expected that with a load of $138 \mu \mathrm{W}$, the still temperature will be $355 \mathrm{mK}$, with a hold time $>36$ hours.

The heat load on the UC stage is dominated by the optical power absorbed by the detector arrays. Note also that there is still a significant radiative load since there is no additional radiation shielding around the UC hardware. From the measured ultracold stage cooling capacity, a heat load of $2.348 \mu \mathrm{W}$ will result in a cold head temperature of approximately $240 \mathrm{mK}$ and a hold time $>15$ days.

The thermal links to the ${ }^{3} \mathrm{He}$ fridge cold heads are made of .004" thick ETP copper foil. From thermal modeling, discussed in more detail below, it was determined that the improvements in conductivity from the use of higher purity foils was unnecessary. The use of thin foils is advantageous in that the finished strap assemblies provide flexibility to allow for movement during thermal contraction. The straps consist of stacks of foil (15 layers for the IC thermal links, 20 layers for the UC wick) electron beam welded to solid ETP copper end pieces. The thermal conductance across e-beam welded joints is similar to that of the bulk material. ${ }^{13}$ Demountable contacts at each end of the strap are made using bolted joints between gold plated surfaces. Gold plating of the surfaces prevents the formation of the oxide layer which would otherwise form on bare copper and degrade the thermal conductance across the joint. ${ }^{14}$ To maximize the conductance across the bolted joints, a number of large screws with high torque are used at each interface to ensure a high clamping force between the surfaces.

To confirm that the design of the heat straps at the sub-kelvin stages did not result in excessive temperature gradients between the IC and UC cold heads and the cooled hardware, a process of thermal modelling was used to determine temperature drops at the various stages of the cooling paths. For these models, a residual resistivity ratio of 90 has been assumed for the ETP copper links, a value that lies at the lower end of the measured range for this type of copper. The conductances of the bolted joints in the cooling paths is based on literature measurements made in the temperature range $300 \mathrm{mK}$ to $4 \mathrm{~K}$, with a scaling factor applied to account for the joints in this design being of larger contact area and made with higher torques. The following paragraphs describe the specific design of the sub-K cooling paths, with reference to the thermal models.

\footnotetext{
${ }^{\S}$ http://www.corning.com/gilbert
} 


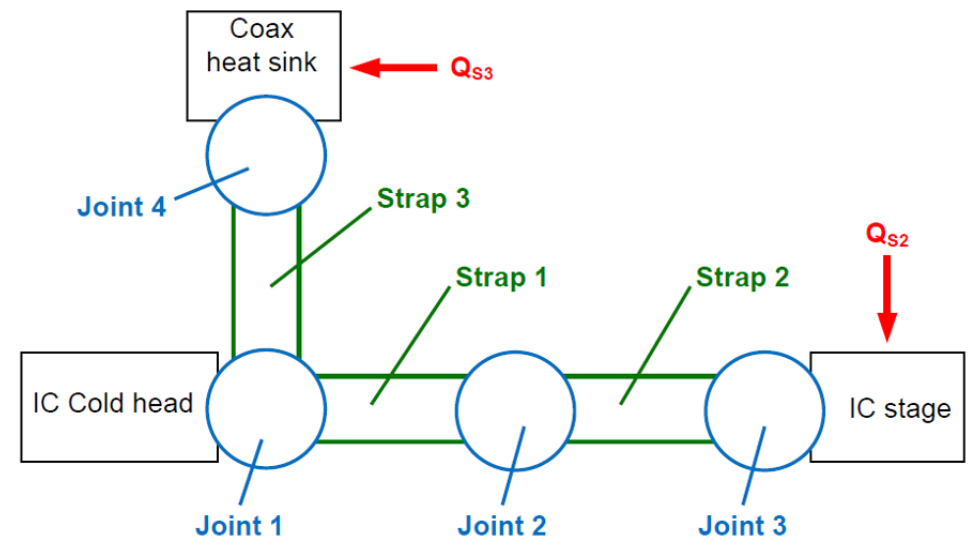

Figure 10: Block diagram of the third stage for thermal modelling. The numbering of joints and straps matches that used in Table 4.

Table 4: Calculated temperature profiles for the IC stage cooling paths. The joint and strap numbering in this table match that in the block diagram in Fig. 11 and the description in the text.

\begin{tabular}{lccc}
\hline Element & $\Delta T / \mathrm{mK}$ & Temperature at warm side $/ \mathrm{mK}$ & Power $/ \mu \mathrm{W}$ \\
\hline IC cold head & - & 355.0 & 138 \\
Joint 1 & 1 & 356.0 & 138 \\
Strap 1 & 3.4 & 359.4 & 61.3 \\
Joint 2 & 0.4 & 359.8 & 61.3 \\
Strap 2 & 0.4 & 360.2 & 61.3 \\
Joint 3 & 0.4 & 360.6 & 61.3 \\
\hline Strap 3 & 4.3 & 360.3 & 76.7 \\
Joint 4 & 0.5 & 360.8 & 76.7 \\
\hline
\end{tabular}

A block diagram of the third (IC) stage thermal model is shown in Fig. 10. The following description of the IC cooling path design references this block diagram. After the demountable joint at the IC cold head (J1), independent straps run to the coax heatsink and to the IC stage inside the magnetic shield. The thermal link to the IC stage transitions from the flexible foil section (strap 1) to a solid ETP copper cold bar (strap 2) via a bolted joint (J2). The use of a solid bar minimizes the risk of a thermal short between the thermal link and the 4-K structures due to thermal contraction during cooling. The solid cold bar attaches to the aluminium IC stage via a bolted joint (J3). The cooling path to the IC coax heatsink also uses a flexible foil section (strap 3), and is attached to the heat sink via a bolted joint (J4). Note that in the this model, it is assumed that there is no temperature drop across the welded joints between the foil straps and the solid copper end pieces.

The predicted temperature profile of the third stage heat straps is summarized in Table 4 . The temperature drop from the IC still to the cold stage is predicted to be $<6 \mathrm{mK}$, dominated by the $\Delta T$ across strap 1 . A similar temperature gradient is predicted between the IC still and the coax heat sink stage, again dominated by the flexible strap. Although these temperature drops could be reduced by increasing the number of foils in the heat strap stacks, the decision was made not to do so. The temperature gradient predicted using this pessimistic value of the copper RRR is acceptable, while measurements of similar copper foil stock from the same supplier has indicated RRR values in the $300-400$ range. ${ }^{15}$

Fig. 11 shows the block diagram for the fourth stage thermal model. The arrangement of the cooling path is similar to that at the third stage, with a demountable bolted joint at the UC still (J1), then a flexible foil strap 


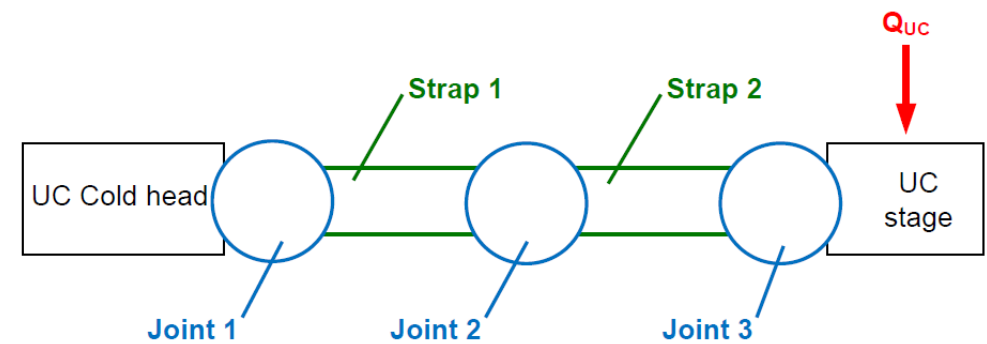

Figure 11: Block diagram of the fourth stage for thermal modelling. The numbering of joints and straps matches that used in Table 5.

Table 5: Calculated temperature profiles for the UC stage cooling paths. The joint and strap numbering in this table match that in the block diagram in Fig. 11 and the description in the text. Fix this, power is too low.

\begin{tabular}{lccc}
\hline Element & $\Delta T / \mathrm{mK}$ & Temperature at warm side $/ \mathrm{mK}$ & Power $/ \mathrm{nW}$ \\
\hline UC cold head & - & 240.27 & 2.348 \\
Joint 1 & 0.02 & 240.29 & 2.348 \\
Strap 1 & 0.23 & 240.52 & 2.348 \\
Joint 2 & 0.02 & 240.54 & 2.348 \\
Strap 2 & 0.23 & 240.77 & 2.348 \\
Joint 3 & 0.02 & 240.79 & 2.348 \\
\hline
\end{tabular}

(strap 1). A bolted joint (J2) connects the foil strap to a solid cold bar (strap 2) that runs through the magnetic shield and the superconducting shield at the IC stage, and connects to the copper UC stage via a further bolted joint (J3). Table 5 summarizes the predicted temperature distribution along the UC cooling path. The predicted $\Delta T$ from the cold head to the stage is $\sim 0.5 \mathrm{mK}$, dominated as at the third stage by the foil strap and the cold finger rather than losses across the bolted joints.

\section{SUMMARY AND CONCLUSIONS}

MUSIC, the Multicolor submillimeter kinetic inductance camera, is a new facility instrument for wide-field millimeter wave imaging at the Caltech Submillimeter Observatory. With a focal plane of antenna-coupled microwave kinetic inductance detectors, a relatively new type of pair-breaking superconducting photon detectors, MUSIC will offer simultaneous imaging in 4 bands with a field of view $>14$ '. This paper has described the design of the four-stage, closed-cycle cryostat for the instrument. Although some of the design criteria proved to be challenging, the final design meets all of the camera requirements. The predicted thermal performance of the system has been modeled and described, and is found to be sufficient for operation of the instrument.

The camera is currently undergoing assembly, integration and verification, and is expected to be deployed to the CSO early in 2011.

\section{ACKNOWLEDGMENTS}

The MUSIC project is supported by NSF grants AST-0705157 to the University of Colorado and AST-0838261 to the Caltech Submillimeter Observatory, NASA grants NNGC06C71G and NNX10AC83G to the California Institute of Technology, the Gordon and Betty Moore Foundation, and the JPL Research and Technology Development Fund. Jack Sayers was supported by a NASA Postdoctoral Program Fellowship, and Nicole Czakon and James Schlaerth by NASA Graduate Student Researchers Program Fellowships. 


\section{REFERENCES}

1. K. D. Irwin, "An application of electrothermal feedback for high resolution cryogenic particle detection," Applied Physics Letters 66, pp. 1998-2000, Apr. 1995.

2. J. A. Chervenak, K. D. Irwin, E. N. Grossman, J. M. Martinis, C. D. Reintsema, and M. E. Huber, "Superconducting multiplexer for arrays of transition edge sensors," Applied Physics Letters 74, p. 4043, June 1999.

3. W. Holland, M. MacIntosh, A. Fairley, D. Kelly, D. Montgomery, D. Gostick, E. Atad-Ettedgui, M. Ellis, I. Robson, M. Hollister, A. Woodcraft, P. Ade, I. Walker, K. Irwin, G. Hilton, W. Duncan, C. Reintsema, A. Walton, W. Parkes, C. Dunare, M. Fich, J. Kycia, M. Halpern, D. Scott, A. Gibb, J. Molnar, E. Chapin, D. Bintley, S. Craig, T. Chylek, T. Jenness, F. Economou, and G. Davis, "SCUBA-2: a 10,000-pixel submillimeter camera for the James Clerk Maxwell Telescope," in Millimeter and Submillimeter Detectors and Instrumentation for Astronomy III., J. Zmuidzinas, W. S. Holland, S. Withington, and W. D. Duncan, eds., Proceedings of the SPIE 6275, July 2006.

4. P. K. Day, H. G. LeDuc, B. A. Mazin, A. Vayonakis, and J. Zmuidzinas, "A broadband superconducting detector suitable for use in large arrays," Nature 425, pp. 817-821, Oct. 2003.

5. P. R. Maloney, N. G. Czakon, P. K. Day, T. P. Downes, R. Duan, J. Gao, J. Glenn, S. Golwala, M. Hollister, H. G. Leduc, B. Mazin, O. Noroozian, H. T. Nguyen, J. Sayers, J. Schlaerth, S. Siegel, J. E. Vaillancourt, A. Vayonakis, P. Wilson, and J. Zmuidzinas, "MUSIC for sub/millimeter astrophysics," these proceedings.

6. J. Schlaerth, N. G. Czakon, P. K. Day, T. P. Downes, R. Duan, J. Gao, J. Glenn, S. Golwala, M. Hollister, H. G. Leduc, B. Mazin, P. R. Maloney, O. Noroozian, H. T. Nguyen, J. Sayers, S. Siegel, J. E. Vaillancourt, A. Vayonakis, P. Wilson, and J. Zmuidzinas, "MKID multicolor array status and results from DemoCam," these proceedings.

7. N. G. Czakon, P. K. Day, T. P. Downes, R. Duan, J. Gao, J. Glenn, S. Golwala, M. Hollister, H. G. Leduc, B. Mazin, P. R. Maloney, O. Noroozian, H. T. Nguyen, J. Sayers, J. Schlaerth, S. Siegel, J. E. Vaillancourt, A. Vayonakis, P. Wilson, and J. Zmuidzinas, "Optimization of MKID noise performance via readout technique for astronomical applications," these proceedings.

8. J. Sayers, N. G. Czakon, P. K. Day, T. P. Downes, R. Duan, J. Gao, J. Glenn, S. Golwala, M. Hollister, H. G. Leduc, B. Mazin, P. R. Maloney, O. Noroozian, H. T. Nguyen, J. Schlaerth, S. Siegel, J. E. Vaillancourt, A. Vayonakis, P. Wilson, and J. Zmuidzinas, "Optics for MUSIC: a New (Sub)millimeter Camera for the Caltech Submillimeter Observatory," these proceedings.

9. J. E. Healey, T. Lindström, M. S. Colclough, C. M. Muirhead, and A. Y. Tzalenchuk, "Magnetic field tuning of coplanar waveguide resonators," Applied Physics Letters 93, p. 043513, July 2008.

10. M. Hollister, H. McGregor, A. Woodcraft, D. Bintley, M. MacIntosh, and W. Holland, "Cryogenic magnetic shielding for SCUBA-2," in Millimeter and Submillimeter Detectors and Instrumentation for Astronomy III., J. Zmuidzinas, W. S. Holland, S. Withington, and W. D. Duncan, eds., Proceedings of the SPIE 7020, Aug. 2008.

11. P. A. R. Ade, G. Pisano, C. Tucker, and S. Weaver, "A review of metal mesh filters," in Millimeter and Submillimeter Detectors and Instrumentation for Astronomy III., J. Zmuidzinas, W. S. Holland, S. Withington, and W. D. Duncan, eds., Proceedings of the SPIE 6275, July 2006.

12. M. C. Runyan and W. C. Jones, "Thermal conductivity of thermally-isolating polymeric and composite structural support materials between 0.3 and 4 K," Cryogenics 48, pp. 448-454, Sept. 2008.

13. A. L. Woodcraft, M. I. Hollister, D. Bintley, F. C. Gannaway, D. C. Gostick, and W. S. Holland, "Thermal design and performance of the SCUBA-2 instrument 1-K and mK systems," Cryogenics 49, pp. 504-513, Sept. 2009.

14. I. Didschuns, A. L. Woodcraft, D. Bintley, and P. C. Hargrave, "Thermal conductance measurements of bolted copper to copper joints at sub-Kelvin temperatures," Cryogenics 44, pp. 293-299, May 2004.

15. M. C. Runyan. Personal communication. 